\title{
Neutron and X-Ray Studies of Advanced Materials Foreword
}

The selection of articles in the special topic "Neutron and X-Ray Studies of Advanced Materials" is based on the materials presented during the TMS 2009 annual meeting in San Francisco, CA, February 15-19, 2009.

The development of ultrabrilliant third-generation synchrotron X-ray sources, together with advances in X-ray optics, has created intense X-ray microbeams, which provide the best opportunities for in-depth understanding of mechanical behavior in a broad spectrum of materials. Important applications include ultrasensitive elemental detection by X-ray fluorescence/absorption and microdiffraction to identify phase and strain with submicrometer spatial resolution. X-ray microdiffraction is a particularly exciting application compared with alternative probes of crystalline structure, orientation, and strain. X-ray microdiffraction is nondestructive with good strain resolution, competitive or superior spatial resolution in thick samples, and with the ability to probe below the sample surface. Moreover, the high-energy X-ray diffraction technique provides an effective tool for characterizing the mechanical and functional behavior in various environments (temperature, stress, and magnetic field).

At the same time, some neutron diffraction instruments constructed mainly for the purpose of engineering applications can be found at nearly all neutron facilities. The first generation-dedicated instruments designed for studying in-situ mechanical behavior have been commissioned and used, and industrial standards for reliable and repeatable measurements have been developed. Furthermore, higher penetration of neutron beams into most engineering materials provides direct measurements on the distribution of various stresses (i.e., types I, II, and III) beneath the surface up to several millimeters, even tens of millimeters for important industrial components.

With X-ray and neutron measurements, it is possible to characterize material behavior at different length scales. It is predicted that the application of these techniques, in combination with theoretical simulations and numerical modeling, will lead to major breakthroughs in materials science in the foreseeable future, which will contribute to the development of materials technology and industrial innovation. Specifically, the use of these techniques provides bulk material properties that further augment new characterization tools including the increased use of atom probe tomography and high-resolution transmission electron microscopy systems. The combination of these techniques greatly assists the material property models that address multi-length-scale mechanisms.

Different applications of diffuse scattering for understanding the fundamental materials properties are illustrated in the articles of Welberry et al., Goossens and Welberry, Campbell, Abe et al., Gilles et al., and Zhang et al. Analysis of thin films and two-dimensional structures is described in the articles of Gramlich et al., Brock et al., Vigliante et al., Kuzel et al., and Davydok et al. Recent advances in the line profile analysis are represented by the the articles of Scardi et al., Ungar et al., and Woo et al. Characterization of modern alloys is presented by the articles of Wollmershauser et al., Eidenberger et al., Garlea et al., Jia et al., Soulami et al., Wilson et al., and Wang et al.

The collected articles are written by different scientific X-ray and neutron research groups. They represent a general trend in the development and application of diffraction techniques all over the world.

Rozaliya I. Barabash

Oak Ridge National Laboratory Oak Ridge, TN 37831-6118

Jaimie Tiley

Air Force Research Laboratory Wright-Patterson AFB, OH 45433

Yandong Wang

Northeastern University Shenyang 110004, People's Republic of China

Peter K. Liaw The University of Tennessee

Knoxville, TN 37996-2200 